\title{
LSTM tabanlı Derin Sinir A ğı ile Ayak Taban Basınç Verilerinden VKİ Durumlarının Sınıflandırılması
}

\author{
Ahmet Ali SÜZEN ${ }^{* 1}$, Ziya YILDIZ ${ }^{2}$, Tuğrul YILMAZ ${ }^{3}$ \\ ${ }^{1}$ Isparta Uygulamalı Bilimler Üniversitesi, Bilgisayar Teknolojileri Bölümü, Isparta \\ ${ }^{2}$ Isparta Uygulamalı Bilimler Üniversitesi, Terapi ve Rehabilitasyon Bölümü, Isparta \\ ${ }^{3}$ Necmettin Erbakan Üniversitesi, Tip Fakültesi, Konya \\ (ORCID: 0000-0002-5871-1652) (ORCID: 0000-0002-8631-9535)( ORCID: 0000-0001-5744-0902)
}

\begin{abstract}
$\ddot{O} z$
Obezite vücut yağ miktarının artması ile çeşitli sorunlara yol açan ve tedavi edilebilen bir hastalıktır. Artan vücut ağırlığı, kas iskelet sistemi üzerinde bozukluklara yol açabilmektedir. Ayak, vücut ağırlığının yere aktarılmasında görevli bir yapıdır. Obezitenin ayağın anatomik yapısını, taban temas yüzey alanını ve basınç değişimlerini etkilendiği görülmüştür. Bu çalışmada Uzun Kısa-Vadeli Hafiza (LSTM) tabanlı derin sinir ağı (DSA) ile bireylerin ölçülen ayak tabanı basınç değerleri ile vücut kitle indeksi (VKI) durumları sınıflandıran bir model geliştirilmiştir. Öncelikle bireyin ayak tabanı ait 12 farklı bölgeye ait basınç değerleri baropodometre ile ölçülmüştür. Ölçüm sonucunda sinir ağının veri kümesinde kullanılacak 13 farklı giriş parametresi elde edilmiştir. Bunun yanında her bireyin VKİ verisi hesaplanarak, bu verilere ait 4 gruptan birine yerleştirilmiştir. Böylelikle modelin öğrenmesi için gerekli veri kümesi oluşturulmuştur. Derin sinir ağında veri kümesi $\% 80$ eğitim $\% 20$ test olarak bölümlenmiştir. Ağın hiper parametrelerin belirlenmesinden sonra eğitim ve test işlemleri gerçekleştirilmiştir. Eğitilen modelin test sonucunda \%93.2 doğruluk elde edilmiştir. Önerilen LSTM model, diğer sınıflandırma algoritmaları ile kıyaslanmıştır. Bunun sonucunda en yüksek performans elde etmiștir. Sonuç olarak önerilen sınıflandırma modeli ile bireyin VKI'sine göre belirlenmiş obezite durumunu, ayak basınç verilerinin sınıflandırılması ile doğrulanmış olmaktadır. Ayrıca LSTM tabanlı derin sinir ağlarının, sınıflandırma işlemlerinde yüksek başarı performansı verdiği gözlemlenmiştir.
\end{abstract}

Anahtar kelimeler: Derin Öğrenme, Uzun-Kısa Süreli Bellek, Sınıflandırma, Vücut Kitle İndeksi.

\section{Classification of BMI States from Foot Base Pressure with LSTM-based Deep Neural Network}

\begin{abstract}
Obesity is a disease that leads to various problems with the increase in the amount of fat in the body. Increased body weight can lead to disorders in the musculoskeletal system. The foot is a structure responsible for transferring body weight to the ground. Obesity has been shown to affect the anatomical structure of the foot, the floor contact surface area and pressure changes. In this study, Long Short-Term Memory (LSTM) based deep neural network (DNN) and individuals measured foot pressure values and body mass index (BMI) states were developed. First of all, the pressure values of the individual's foot base 12 different regions were measured by baropodometer. As a result of the measurement, 13 different input parameters were obtained to be used in the neural network data set. In addition, BMI data of each individual were calculated and placed in one of 4 groups of this data. Thus, the necessary data for the model to learn was created. In the deep neural network, data were divided into $80 \%$ of the training and 20\% of the testing. After the determination of hyper-parameters of the network, training and testing procedures were performed. In the test result of the trained model was achieved $93.2 \%$ accuracy. As a result, the proposed classification model is verified by the classification of the foot pressure data according to the status of obesity determined by the individual's BMI. In addition, LSTM-based deep neural networks have been observed to provide high accuracy performance in classification operations.
\end{abstract}

Keywords: Body Mass Index, Classification, Deep Learning, Long Short-Term Memory.

"Sorumlu yazar:ahmetsuzen@isparta.edu.tr

Geliş Tarihi: 14.03.2019, Kabul Tarihi: 11.07.2019 


\section{Giriş}

Fosil ayak izleri incelendiğinde dik yürüyen, iki ayaklı bir yaşayışa rastlanmıştır [1]. Ayak görevleri göz önüne alındığında destek yüzeyi oluşturmak, şokları absorbe etmek, harekete ve zemine uyum göstererek ve rijit bir kaldıraç görevi üstlenmektir. Ayaktaki anormal yapı ve mekaniklerindeki değişim bireyin yaralanma riskini arttırabilir [2,3]. Obezite adipoz doku birikimiyle vücut yağ kitlesinin, yağsız vücut kitlesine oranla artmasıyla oluşan karakterize kronik bir hastalıktır. Obezitenin sınıflandırmasında vücut kitle indeksi (VKİ) en çok kullanılan yöntemdir. VKİ, kilogram cinsinden vücut ağırlığının, metre cinsinden vücut uzunluğunun karesine oranlanması ile bulunur [4,5]. OECD ülkeleri arasında yapılan araştırmada obezite \%22,3 oranla Türkiye'de 7. sırada yer almaktadır [6]. Obezite ile ayak taban basıncı ve yüzey alanında değişimlerin olduğu görülmüştür. Baropodometre cihazı ayak tabanından basıncı ve yüzey alanını ölçmektedir. Baropodometreler bir platform üzerine basınç ve alan sensörlerin yerleştirilmesi ile oluş̧urulur. Sensörden alınan veriler geliştirme kartlarında işlenerek basınçlar farklı renk ve tonlar ile görsel ortama aktarılır [7].

Obezite ile ayağın taban kısmında temas alanında ve basıncında pozitif korelasyon görülmüştür [8]. Çalışmalarda ayağın orta iç kısmı, başparmak altı ve topukta basıncı ve temas alanının istatistiki olarak anlamlı ilişki bildirilmiştir $[9,10]$. Obezite ile ayak anatomik yapısında bozukluklar meydana gelmektedir. Bu bozukluklar sonrası diğer eklemlerde de kronik problemlere yol açıldığı görülmüştür. Ayrıca obezitenin bağları fazla germesi sonucunda duyu kaybı olacağı ve statik duruşta artan bir bozulma olacağı düşünülmektedir [11,12].

Verinin oldukça fazla olduğu günümüzde, verilerden sonuçlar çıkarmak, sınıflandırmak veya gelecek ile ilgili tahminlerde bulunmak için derin öğrenme oldukça fazla kullanmaktadır [13]. Özellikle çok fazla verinin olduğu problemlerin çözümünde kullanılan derin öğrenme algoritmalarının yüksek başarı performansı sağladığı görülmektedir [14,15]. Temel olarak derin öğrenme çoklu soyut yapısı ile çok katmanlı bir yapay sinir ağı modelidir [16]. Derin öğrenme; konvolüsyonel sinir ağı (Convolution Neural Network-CNN), Tekrarlayan Sinir Ağ 1 (Recurrent Neural Network-RNN), Uzun Kısa Süreli Bellek Ağları (Long Short Term Memory - LSTM), Sınırlı Boltzmann makineleri (Restricted Boltzmann Machines-RBM), Oto-kodlayıcılar (Autoencoders-AE) ve Derin veya yığınlı oto-kodlayıcılar (Deep/Stacked Autoencoder-DAE) mimarilerinden oluşmaktadır [17].

Derin öğrenme algoritmalarından birisi olan LSTM, ilk olarak RNN olarak tanıtılan ve daha sonra araştırmacılar tarafından RNN'in eksikliklerine göre değiştirilen bir DSA mimarisidir [17]. LSTM ağların özellikle çok değişkenli sınıflandırma ve tahmin problemleri üzerinde başarılı sonuçlar verdiği görülmektedir [18-20]. Önerilen model için 13 farklı parametre bulunduğundan ve bireyin belirli bir zaman serisinde VKİ durumların sınıflandırılabileceği nedeni ile LSTM ağı seçilmiştir. Bu çalışmada bireylerin ayak basınç değerlerin ölçülmesinden oluşan veri kümesi ile VKİ durumlarının sınıflandırılmasını sağlayan bir sinir ağı modellenmiştir. Modelin geçerliliği için farklı sınıflandırma algoritmaları ile kıyaslama yapılmış ve önerilen modelin en yüksek doğruluğa sahip olduğu belirlenmiştir.

Çalışmanın bu bölümünden sonra öncelikle uzun-kısa süreli bellek derin öğrenme modeli anlatılmıştır. Uzun kısa vadeli bellek modelinden sonra ilk olarak önerilen sınıflandırma modelinin veri kümesinde kullanılan verilerin hazırlanması, eğitim ve test için bölünmesi anlatılmaktadır. Önerilen model bölümünde sınıflandırma işlemi için geliştirilen modelin katmanları ve hiper-parametreler belirlenmiştir. Çalışmanın son kısmında LSTM sinir ağının eğitilmesi ve test süreçleri gösterilmiştir. Buna bağlı olarak elde edilen sonuçlar değerlendirilmiştir.

\section{Uzun Kısa Vadeli Bellek (LSTM)}

Uzun kısa vadeli bellek modeli, 1997'de Hochreiter ve Schmidhuber tarafindan ortaya atılan bir tekrarlayan RNN sinir ağı olarak bilinmektedir [21]. Geleneksel RNN sinir ağlarının eğitilmesinde yaşanan sorunlar LSTM'de bütünüyle giderilmiştir. LSTM, hafıza geçişli mekanizması ile uzun vadeli bağımlılıkları öğrenebildiği için sıralı veya zaman serisi problemlerde oldukça fazla kullanılmaktadır [22]. Temel LSTM mimarisi giriş (Denklem 1), çıkış (Denklem 4), unut kapıları (Denklem 2) ve hafiza hücrelerinden (Denklem 3) oluşmaktadır. Sinir ağının kapı ve hücrelerine ait işlem fonksiyonları aşağıda verilmiştir. Ayrıca temel LSTM hücre yapısına ait diyagram görüntüsü Şekil 1'de gösterilmiştir. 


$$
\begin{aligned}
& \mathrm{i}_{\mathrm{t}}=\sigma\left(\mathrm{w}_{\mathrm{xi}} \mathrm{x}_{\mathrm{t}}+\mathrm{w}_{\mathrm{hi}} \mathrm{h}_{\mathrm{t}-1}+\mathrm{w}_{\mathrm{ci}} \mathrm{c}_{\mathrm{t}-1}+\mathrm{b}_{\mathrm{i}}\right) \\
& \mathrm{f}_{\mathrm{t}}=\sigma\left(\mathrm{w}_{\mathrm{xf}} \mathrm{x}_{\mathrm{t}}+\mathrm{W}_{\mathrm{hf}} \mathrm{h}_{\mathrm{t}-1}+\mathrm{w}_{\mathrm{cf}} \mathrm{c}_{\mathrm{t}-1}+\mathrm{b}_{\mathrm{f}}\right) \\
& \mathrm{c}_{\mathrm{t}}=\mathrm{f}_{\mathrm{t}} \odot \mathrm{c}_{\mathrm{t}-1}+\mathrm{it} \odot \tanh \left(\mathrm{w}_{\mathrm{xc}} \mathrm{x}_{\mathrm{t}}+\mathrm{w}_{\mathrm{hc}} \mathrm{h}_{\mathrm{t}-1}+\mathrm{b}_{\mathrm{c}}\right) \\
& \mathrm{o}_{\mathrm{t}}=\sigma\left(\mathrm{w}_{\mathrm{xo}} \mathrm{x}_{\mathrm{t}}+\mathrm{w}_{\mathrm{ho}} \mathrm{h}_{\mathrm{t}-1}+\mathrm{w}_{\mathrm{co}} \mathrm{c}_{\mathrm{t}}+\mathrm{b}_{\mathrm{o}}\right)
\end{aligned}
$$

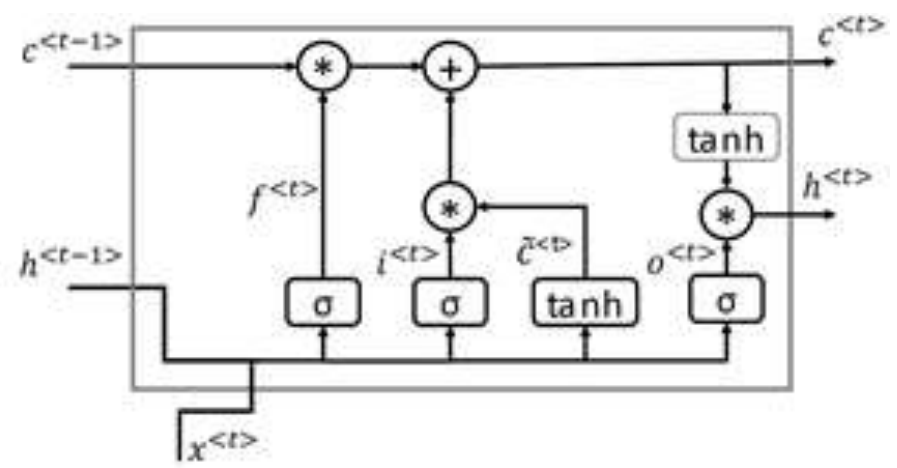

Şekil 1.Temel LSTM blog diyagramı [23].

Burada $i, o, f$ ve c terimleri sırasıyla giriş kapısı, çıkış kapısı, unut kapısı ve hafiza hücrelerini ifade etmektedir. LSTM algoritmaları özellikle konuşma, metin işleme, müzik, zaman serisi tahmin, sınıflandırma uygulamalarında LSTM mimarileri kullanılmış ve başarılı sonuçlar alınmıştır [24-26].

\section{Verilerin Hazırlanması}

\subsection{Verilerin Ölçülmesi}

Ölçüm verileri herhangi bir sağlık problemi olmayan çeşitli VKİ sahip 18-59 yaş 108 bireyden alınmıştır. Verilerin toplanması, Şekil 2-a' da görüldüğ̈̈ gibi çıplak ayakla baropodometre cihaz platformu üzerinde çift ayak duruşu esnasında gerçekleşmiştir. Ayaklar arasında bir mesafe düzenleyici konulmuştur. Bu düzenleyici sayesinde ayak tabanı ön-orta-arka ayak olarak üçe ve buralarda iç-dış kısım olarak bölümlere ayrılmıştır. Her iki ayak için Şekil 2-b'de verilen toplam 12 bölgenin basınç verileri ve toplam temas alanı dikkate alınmıştır.

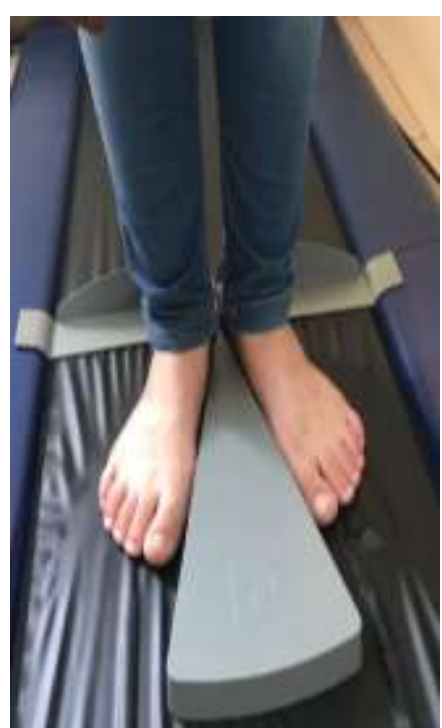

(a)

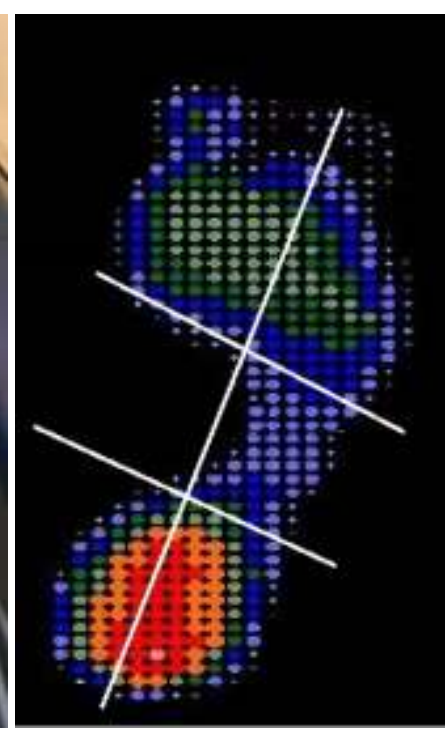

(b)

Şekil 2. (a) Ayak plantar bölgesini 6 parçaya bölünmesini (b) Ölçümlerde ayağın yerleştirilmesi

Sınıflandırma için kullanılacak VKİ grupları, bireylerden boy, kilo ve yaş bilgileri alındıktan sonra Dünya Sağlık Örgütünün belirlediği ve Tablo 1'de verilen şartlara göre belirlenmiştir. 
Tablo 1. VKİ değerine göre gruplama şartlar

\begin{tabular}{|l|l|}
\hline Şart & Grup \\
\hline$<18.5$ & Zaylf \\
$18.5 \leq$ ve $\leq 24.9$ & Normal \\
$25 \leq$ ve $\leq 29.9$ & Kilolu \\
$30 \leq$ & Obez \\
\hline
\end{tabular}

\subsection{Veri Ön Hazırlık}

Bireylerden ölçümü yapılan ve alınan (yaş, boy, kilo) 26 noktadan LSTM sinir ağında kullanılacak parametrelerin seçilmesi gerekmektedir. $\mathrm{Bu}$ noktada varolan veri gruplarının VKİ durumları incelenmiştir. VKİ değişimlerinde değeri değişmeyen sütunlar veri kümesinden çıkartılmıştır. Sonuç olarak veri kümesinde bulunan 26 parametre, 13 bağımsız 1 bağımlı parametreye indirgenmiştir. Tablo 2 'de eğitimde ve testte kullanılan veri kümesindeki verilerin parametre isimleri ve tanımlamaları verilmiştir.

Tablo 2. Veri kümesindeki parametre ve tanımları

\begin{tabular}{ll}
\hline Değişken & Tanım \\
\hline YAS & Bireyin yaş1 \\
LOL & Sol ön ayak lateral bölgede tepe basınç değeri \\
LOM & Sol ön ayak medial bölgede tepe basıç değeri \\
ROM & Sağ ön ayak medial bölgede tepe basıç değeri \\
ROL & Sağ ön ayak lateral bölgede tepe basınç değeri \\
LORL & Sol orta ayak lateral bölgede tepe basıç değeri \\
LORM & Sol orta ayak medial bölgede tepe basınç değeri \\
RORL & Sağ orta ayak lateral bölgede tepe basıç değeri \\
RORM & Sağ orta ayak medial bölgede tepe basıç değeri \\
LAL & Sol arka ayak lateral bölgede tepe basınç değeri \\
LAM & Sol arka ayak medial bölgede tepe basıç değeri \\
RAL & Sağ arka ayak lateral bölgede tepe basıç değeri \\
RAM & Sağ arka ayak medial bölgede tepe basıç̧ değeri \\
VKI_Durum & Sinıflandırma türü \\
\hline
\end{tabular}

Normalleştirme işlemi ham verilere uygulanarak, eğitim için gerekli veri kümesinin son şeklinin oluşturulmasını sağlar. Normalleştirme yapmak için Min-Max, Medyan, Sigmoid ve Z-Score gibi teknikler kullanılmaktadır. Bu tekniklerden en yaygın olarak kullanılanı min-max normalizasyonudur (Denklem 5).

$\mathrm{z}=\frac{x-\min (\mathrm{x})}{\max (x)-\min (x)}$

\section{Model}

Modelin eğitilmesinde ağılık matrisleri sıfıra yakın değerler ile başlatıldı. Öğrenme oranı ise başlangıçta $\alpha=0.01$, eğitim tur değeri 10 'dan sonra $\alpha=0.001$ olarak güncellenmiştir. Modelin eğitilmesi için gerekli hiper parametreler Tablo 3'de gösterildiği gibi belirlenmiştir.

Tablo 3. Modelin hiper ve standart parametreleri

\begin{tabular}{|l|l|}
\hline Hiper parameter ve diğer parametreler & Değer \\
\hline Eğitim Verisi (train data) & $87(80 \%)$ \\
Test Verisi (test data) & $21(20 \%)$ \\
Giriş (input) & 13 \\
Batch Boyutu (batch size) & 8 \\
Eğitim Sayısı (epoch) & 100 \\
Öğrenme Hızı (learning rate) & 0.001 \\
Seyreltme Değeri (dropout) & 0.5 \\
Katman (layer) & 3 \\
Çıkış (output) & 1 \\
\hline
\end{tabular}


Eğitilmiş modelin gizli katmanın çıkışında sınıflandırma Denklem 6'da formüle edilen softmax çoklu sınıflandırma fonksiyonu kullanılarak oluşturulmuştur. Softmax fonksiyonu çıkışta her bir girdinin bir sınıfa ait olma durumunu [0,1] arasında çıktı üreterek göstermektedir.

$$
\frac{\partial}{\partial_{z_{i}}} \sigma(x)=\frac{\partial}{\partial_{z_{i}}} \frac{e^{x_{i}}}{\sum_{k=1}^{k} e^{x_{k}}} \quad \text { for } i=1, \ldots \ldots . . .
$$

Eğitilen sinıflandırma modelinin doğruluğunu ölçmek için ortalama kare hata (MSE - Mean Square Error) kullanılmıştır. Denklem 7'de gösterilen $a_{i}$, tahmin verisini $p_{i}$, ölçülen veriyi temsil etmektedir.

$$
M S E=\frac{1}{n} \sum_{i=1}^{n}\left(a_{i}-p_{i}\right)^{2}
$$

Önerilen model, Windows 10 Pro işletim sistemi üzerinde Intel I7 2.9 GHZ işlemci, 12 GB RAM ve NVIDIA 940MX grafik kartına sahip donanımda geliştirilmiştir. Eğitilen modelin her eğitim turuna ait doğruluk değerleri eğitim ve test olarak Şekil 3'de gösterilmiştir. Şekilden de görüldüğü gibi modelin eğitimi, eğitim turu sayısı 60 'den sonra tamamlanmıştır. Ayrıca eğitim ve teste ait toplam performansı Tablo 4'de gösterilmektedir.

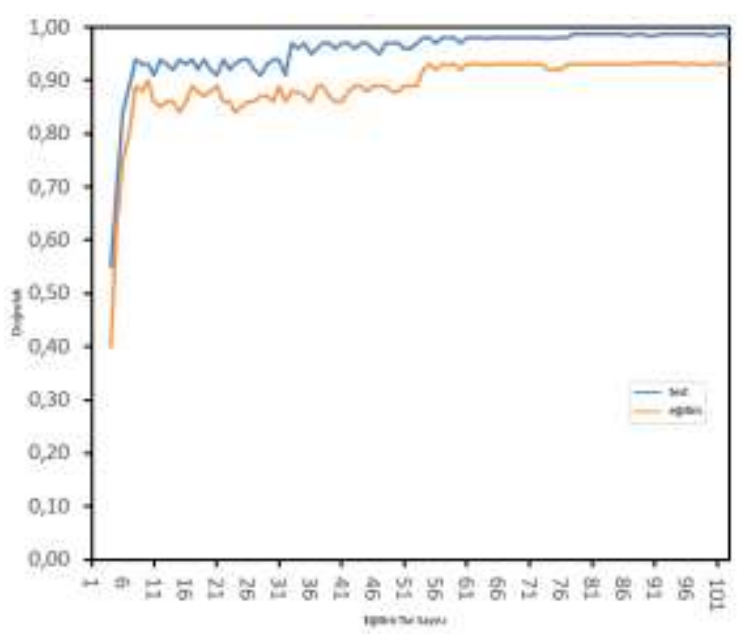

Şekil 3. Modelin her eğitim turuna ait eğitim ve test doğruluk grafiği

Tablo 4. Eğitilen Modelin Performans Değerleri

\begin{tabular}{lll}
\hline Veri Kümesi & Doğruluk (\%) & Kayıp \\
\hline Eğitim & $\% 98.8$ & 0.0825 \\
Test & $\% 93.2$ & 0.5230 \\
\hline
\end{tabular}

Modelin çıkışında 4 farklı sınıflandırma yapılmıştır. Bu sınıflandırmaya ait karışıklık matrisi Tablo 5'de verilmiştir. Zayıf kişilerin ayak yapılar diğerlerine göre farklı olduğu ölçüm sonuçlarında görülmekteydi. Karışıklık matrisine bakıldığında zayıf kişilerin sınıflandırılmasında başarı oranının daha düşük, diğer sınıflandırmalarda kabul edilebilir değerlerde olduğu görülmektedir.

Tablo 5.Ĕgitilen modelin karıșıklık matrisi

\begin{tabular}{|l|c|c|c|c|}
\cline { 2 - 5 } \multicolumn{1}{c|}{} & $\mathrm{Z}$ & $\mathrm{N}$ & $\mathrm{K}$ & $\mathrm{O}$ \\
\hline Zayıf & 89,5 & 8,2 & 2,3 & 0 \\
\hline Normal & 2,1 & 95,4 & 2,5 & 0 \\
\hline Kilolu & 0 & 4,6 & 93,1 & 2,3 \\
\hline Obez & 0 & 1,2 & 4,0 & 94,8 \\
\hline
\end{tabular}

Önerilen LSTM'den elde edilen sonuçların ne kadar başarılı olduğu belirlememek için aynı veri kümesi ile sınıflandırmada kullanılan farklı öğrenme algoritmaları kullanılmıştır. Bu çalışmaya 
kıyaslama için Karar Ağacı Regresyonu, Lojistik Regresyon, Destek Vektör Makinesi ve Naive Bayes algoritmaları seçilmiştir. Aynı test ortamında gerçekleşen kıyaslama sonucu Tablo 6' da verilmiştir. Buna göre önerilen LSTM model, kullanılan veri kümesinde en yüksek doğruluğu elde ettiği görülmüştür.

Tablo 6. Sınıflandırma yöntemlerinden elde edilen sonuçların kıyaslanması

\begin{tabular}{|l|l|}
\hline Model & Doğruluk (\%) \\
\hline Karar Ağacı Regresyonu & 76,2 \\
\hline Lojistik Regresyon & 87,8 \\
\hline Destek Vektör Makinesi & 88,6 \\
\hline Naive Bayes & 91,1 \\
\hline LSTM & 93,2 \\
\hline
\end{tabular}

\section{Sonuç}

$\mathrm{Bu}$ çalışmada LSTM derin sinir ağları ile bireylerin ölçülen ayak tabanı basınç değerleri ile VKİ durumları sınıflandıran bir model önerilmiştir. Sınıflandırma modelinin veri kümesi, bireyin ayak tabanında 12 farklı bölgeye ait basınç değerleri baropodometre ile ölçülerek oluşturulmuştur. Modelin eğitilmesinde kullanılan veri kümesi \%80 eğitim ve \%20 test olarak bölümlenmiştir. LSTM modelinin eğitiminde \%98.8, testinde ise \%93.2 doğruluk elde edilmiştir. Çalışmadan elde edilen sonuçların başarısını test etmek için model, sınıflandırmada kullanılan geleneksel algoritmalar ile karşılaştırılmıştır. Karş1laştırmanın sonucunda LSTM modelin en yüksek doğruluğa sahip olduğu görülmüştür. Modelden elde edilen sonuçlara göre ayak basınç verileri parametreleri kullanılarak LSTM sinir ağı ile yapılan sınıflandırmanın VKİ 'ye göre yapılan sınıflandırma ile uyumluluk sağladığı görülmüştür. Böylelikle VKİ durumların belirlenmesinde ayak basınç değerlerinde kullanılabileceği öngörülmektedir.

\section{Kaynaklar}

[1] Ozansoy F. 1969. Türkiye Pleistosen fosil insan ayak izleri. Maden Tetkik ve Arama Dergisi, 72 (72).

[2] Uygur Ş.F. 1992. Ayak Deformite ve Ortezleri. Volkan Matbaacilık, 113-127s, Ankara.

[3] Franco A.H. 1987. Pes Cavus and Pes Planus. Analyses and treatment. Physical Therapy, 67 (5): 688-694.

[4] Ergün A., Erten F. 2004. Öğrencilerde Vücut Kitle İndeksi ve Bel Çevresi Değerlerinin İncelenmesi. Ankara Üniversitesi Tip Fakültesi Mecmuas1, 57 (2): 57-61.

[5] İslamoğlu Y., Koplay M., Sunay S., Açıkel M. 2008. Obezite ve Metabolik Sendrom. Tıp Araştırma Dergisi, 6 (3): 168-174.

[6] Çukur A.; Arıtı E.İ. 2017. Obezite Vergilerinin Obezite ile Mücadelede Yeri: Türkiye İçin Bir Değerlendirme. Sayıştay Dergisi, 106s.

[7] Rosário J.L.P. 2014. A Review of the utilization of baropodometry in postural assessment. Journal of Bodywork and Movement Therapies, 18 (2): 215-219.

[8] Tomankova, K., Pridalova, M., Svoboda, Z., Cuberek, R., 2017. Evaluation of Plantar Pressure Distribution in Relationship to Body Mass Index in Czech Women During Walking. Journal of the American Podiatric Medical Association, 107 (3): 208-214.

[9] Fabris S.M., Valezi A.C., Fabris De Souza S.A., Faintuch J., Cecconello I., Pedroni M. 2006. Computerized baropodometry in obese patients. Obesity Surgery, 16 (12): 1574-1578.

[10] Butterworth P.A., Urquhart D.M., Landorf K.B., Wluka A.E., Cicuttini F.M., Menz H.B. 2015. Foot posture, range of motion and plantar pressure characteristics in obese and non-obese individuals. Gait and Posture, 41 (2): 465-469.

[11] Golano P., Fariñas O., Sáenz, I. 2004. The anatomy of the navicular and periarticular structures. Foot and Ankle Clinics, 9 (1): 1-23.

[12] Butterworth P.A., Landorf K.B., Gilleard W., Urquhart D.M., Menz H.B. 2013. The association between body composition and foot structure and function: a systematic review. Obesity Comorbidity, 15 (4): 348-357.

[13] Kızrak M.A., Bolat B. 2018. Derin Öğrenme ile Kalabalık Analizi Üzerine Detaylı Bir Araştırma. Bilişim Teknolojileri Dergisi, 11 (3): 263-286. 
[14] Shu X., Tang J., Qi G.J., Liu W., Yang J. 2018. Hierarchical Long Short-Term Concurrent Memory for Human Interaction Recognition. arXiv preprint arXiv:1811.00270.

[15] Raj H., Weihong Y., Banbhrani S.K., Dino S.P. 2018. LSTM Based Short Message Service (SMS) Modeling for Spam Classification. In Proceedings of the 2018 International Conference on Machine Learning Technologies, 76-80.

[16] Ergen T., Kozat S.S. 2017. Online Training of LSTM Networks in Distributed Systems for Variable Length Data Sequences. IEEE transactions on neural networks and learning systems, 29 (10): 5159-5165.

[17] Şeker A., Diri B., Balık H.H. 2017. Derin Öğrenme Yöntemleri ve Uygulamaları Hakkında Bir İnceleme. Gazi Mühendislik Bilimleri Dergisi, 3 (3): 47-64.

[18] Zhang T., Song S., Li S., Ma L., Pan S., Han L. 2019. Research on Gas Concentration Prediction Models Based on LSTM Multidimensional Time Series. Energies, 12 (1): 161, doi.org/10.3390/en12010161.

[19] Yildirim Ö., Baloğlu U.B., Tan R., Ciaccio E., Acharya R. 2019. A new approach for arrhythmia classification using deep coded features and LSTM networks Computer Methods and Programs in Biomedicine, 176: 121-133.

[20] Xiao L., Wang G., Zuo Y. 2018 Research on Patent Text Classification Based on Word2Vec and LSTM, 2018 11th International Symposium on Computational Intelligence and Design (ISCID), Hangzhou, China, 71-74.

[21] Hochreiter S., Schmidhuber J. 1997. Long short-term memory. Neural Computation, 9 (8): 1735 1780.

[22] Ahmadi N., Constandinou T., Bouganis C., 2019. Decoding Hand Kinematics from Local Field Potentials Using Long Short-Term Memory (LSTM) Network. 9th International IEEE EMBS Conference on Neural Engineering (NER 2019), 1-5s.

[23] Samui S., Chakrabarti I., Ghosh S.K., 2018. Tensor-Train Long Short-Term Memory for Monaural Speech Enhancement. arXiv preprint arXiv:1812.10095.

[24] Graves A., Schmidhuber J. 2005. Framewise phoneme classification with bidirectional LSTM and other neural network architectures. Neural Networks, 8 (5): 602-610.

[25] Graves A.M., Hinton G. 2013. Speech recognition with deep recurrent neural networks, in 2013 IEEE International Conference on Acoustics, Speech and Signal Processing, 6645-6649.

[26] Fernández S., Graves A., Schmidhuber J. 2007. An Application of Recurrent Neural Networks to Discriminative Keyword Spotting. in International Conference on Artificial Neural Networks, 220-229. 